\title{
El efecto de la dependencia-independencia de campo en la formación de recuerdos falsos
}

Field dependence-independence effect on false memories.

Encarnación Bastida Navarro ${ }^{1}$

(1) Universidad Autónoma de Madrid, Madrid, España.

Email correspondencia: Nani.Bastida.Navarro@gmail.com

\section{Resumen}

El presente estudio examina el fenómeno de las memorias falsas de estímulos pictóricos en relación con un estilo cognitivo: la dependenciaindependencia de campo. Se presentó a los participantes la imagen de un dormitorio infantil dentro del cual habia elementos que se clasificaban dentro tres categorias distintas (juguetes, papelería y muebles) y otros elementos que ejercian de distractores (ej. ropa), con la intención de ver si producian más memorias falsas en los elementos de esas tres categorias que en otros objetos de los que no bubiera cantidad suficiente para agruparlos como categorias definidas. Asi mismo se quería comprobar si la presentación de los elementos dentro de un contexto determinado suscitaba el esquema "dormitorio infantil", afectando al recuerdo. Se comparó el resultado en la prueba de memoria con la dependencia e independencia de campo de los participantes, encontrando que, efectivamente, habia relación entre ambos constructos: los independientes de campo resultaron ser menos vulnerables que los dependientes de campo a generar falsas memorias en el campo visual.

Palabras Clave: Memorias falsas; Dependencia-Independencia de Campo; Categorías; Esquema.

\begin{abstract}
This study examines the phenomenon of false memories of pictorial stimuli in relation to a cognitive style: field dependence-independence. Participants were presented the image of a child's bedroom in which there were elements that were classified into three categories (toys, stationery and furniture) and other elements that worked as distractors (eg. clothing). The purpose was seeing if they produced more false memories in the elements of these three categories than in other objects that are not enough to group as defined categories. Also we wanted to test whether the presentation of the elements within a given context raised the schema "children's room", affecting the memory. The result was compared in the memory test with field dependence-independence of the participants, and found that, indeed, there was relationship between the two constructs: independent field were less vulnerable than field dependent to generate false memories in the visual field.
\end{abstract}

Keywords: False memories; Field dependence-independence; Categories; Schema.

Con el agradecimiento a la colaboración de doña Matilde Ortega Maestre.

Fecha de envío: 18/02/2015

Fecha de aceptación: 11/06/2016 


\section{INTRODUCCIÓN}

Según el Proyecto Inocencia, a fecha de 27 de abril de 2016, más del 70\% de las condenas a inocentes en Estados Unidos se deben a errores de memoria de las víctimas o los testigos, que identificaban con una total certeza al acusado.

La memoria humana no es una grabación perfecta de lo que vivimos, comete errores debido a sesgos, sugestiones y olvidos entre otros. Y, en ocasiones, esos errores de la memoria tienen consecuencias tan graves como hacer que un inocente acabe en la cárcel; o insignificantes, al menos en comparación, como olvidar una fecha de cumpleaños o dónde hemos dejado las llaves. De hecho, la gran mayoría de fallos de memoria pasan desapercibidos en nuestro día a día: no recordamos qué desayunamos hace dos semanas, ni cuántos coches rojos nos hemos cruzado viniendo hasta aquí. Esto es porque estamos diseñados para consolidar en nuestra memoria lo relevante y descartar lo poco significativo en pos de un mejor funcionamiento, lo cual nos lleva a ser a veces poco fiables en la recuperación de los recuerdos.

Uno de estos errores son las memorias falsas, que consisten en recordar eventos que nunca sucedieron, o recordarlos de forma distinta a como ocurrieron en realidad. Este fenómeno ha sido estudiado, entre otros motivos, por su aplicación en los estudios sobre testimonio, por la relevancia que tiene, como comentábamos al principio, saber discernir un recuerdo verdadero de uno falso o alterado debido a las graves consecuencias que esto puede acarrear.

La psicología ha de proporcionar investigaciones éticas y que sirvan al bienestar social. Por este motivo, es una cuestión relevante el estudio de variables que puedan ayudarnos a discriminar un buen testigo de uno poco fiable y, a nivel más general, aportar más luz al campo de las memorias falsas.

Esta investigación gira en torno a las memorias falsas en relación a las categorías asociadas, que consisten en que, mediante la presentación de elementos relacionados entre sí por formar parte de la misma categoría, se da lugar el recuerdo falso de otros elementos de la misma categoría no presentados. Una de las investigaciones que ha influenciado en este trabajo ha sido el estudio de Hintzman (1988).
Hintzman presentaba a los participantes una lista con numerosas categorías de palabras cotidianas desordenadas, y de cada categoría presentaba 1, 3 ó 5 palabras. Por ejemplo, de la categoría material de lectura los posibles ítems serían: folleto, cómic, periódico, catálogo, etc. Posteriormente pasaba a los sujetos una prueba de reconocimiento en la que se hallaban las palabras que habían estudiado, como periódico o folleto, y también otras que no habían estudiado previamente pero que eran de la misma categoría como panfleto o revista. Con esto, el investigador trataba de comprobar si, por tratarse de palabras de la misma categoría, el lector cometería el error de memoria de creer recordar las palabras no presentadas previamente. Sin embargo, los resultados mostraron que la tasa de errores no era tan significativa como la de aciertos, aunque sí descubrió que, cuando se aumentaba el número de ítems de cada categoría, aumentaba el número de falsas alarmas mucho más que el de aciertos. Es decir, cuánto más se exigía a la memoria más probable era cometer errores.

Por otra parte, los estudios de Strack y Bless (1994) y Fisher y Sloutsky (2004) demostraron que era indiferente mostrar imágenes que palabras para obtener falsas memorias, incluso utilizando dibujos como es el caso de esta investigación.

El punto de originalidad de la presente investigación reside en poner en relación el uso de categorías asociadas en imágenes para obtener memorias falsas con un estilo cognitivo: la dependencia-independencia de campo.

La dependencia-independencia de campo (DIC) es una aptitud perceptivo-analítica que se manifiesta a través de todo el funcionamiento perceptivo del individuo (Witkin \& Goodenough, 1985; Witkin, Oltman, Raskin \& Karp, 1987). Puede interpretarse como la capacidad para aislar un elemento con respecto al campo organizado en el que se halla inmerso. Dicho de otra manera, la independencia de campo es la capacidad que nos ayuda a encontrar las llaves cuando las perdemos.

Una persona dependiente de campo, cuando observa su entorno, está influenciada por la organización del campo circundante y percibe los componentes como algo difuso. Un sencillo ejemplo: cuando un dependiente de campo observa un bosque, sólo puede ver el bosque. Su mirada hace barridos grandes y holísticos en los cuales es difícil precisar los detalles o analizar por separado las cosas. Por 
otro lado, un independiente de campo percibe la realidad dividida en sus componentes; tiene una mirada mucho más analítica, lo que, en ocasiones, le dificulta la visión global. Un independiente de campo en un bosque sólo vería los árboles, pero no el bosque que forman.

La distribución de este estilo perceptivo no se trata de una clasificación dicotómica, las personas no son simplemente dependientes de campo (DC) o independientes de campo (IC), sino que es un continuo como lo puede ser, por ejemplo, la altura o la inteligencia, por lo que la denominación es relativa. Witkin (1987) consideró que la DIC se manifestaba en la población según la distribución normal, siendo ambos estilos cognitivos los extremos de la campana de Gauss por lo que sólo habría en la población un $15 \%$ de cada uno de ellos.

Desde que Witkin y su equipo colaborador comenzaran a estudiar este estilo cognitivo a finales de los años cuarenta, varios autores han mostrado su interés en él para ponerlo en relación con otras muchas variables. Entre ellos, se debe destacar para el presente estudio a Arnau Grass, Mena y Beltrán (1992), quienes lo pusieron en relación con el procesamiento de la información visual, defendiendo que los DC utilizan estrategias más holísticas y perciben el entorno de manera global y responden a los estímulos como un todo, mientras que los IC se muestran más analíticos y tienden a responder a los ítems en función de sus rasgos locales.

Usando como base las conclusiones de estos estudios, en la presente investigación se decidió que la prueba que se pasaría a los participantes, además de tener un formato visual, sería un dibujo de un dormitorio que contendría en su interior los ítems de las distintas categorías repartidos aleatoriamente, en lugar de presentarlos descontextualizados y en serie. El dormitorio suponía una representación sencilla con la que todos los participantes estarían familiarizados y que permitiría poner a prueba el estilo cognitivo DIC. Según esta base teórica, era de esperar que los participantes IC serían capaces de observar de forma más exhaustiva los elementos de la imagen, mientras que los DC se perderían en el "todo" y tendrían más dificultades para visualizar los elementos por separado.

Sin embargo, al recurrir como procedimiento a la imagen de un dormitorio y elegir como ítems elementos que, en mayor o menor medida, podrían formar parte de él (como por ejemplo una cama o un osito de peluche), en lugar de elementos que no encajaran en ese contexto (como un semáforo o un ataúd), los participantes se verían influenciados por los esquemas mentales.

Cada vez que percibimos algo, nuestra mente, en un intento de hacer la tarea más sencilla o rápida, conecta los datos perceptivos con alguna otra cosa que ya tengamos asimilada. Como dijo Bartlett (1932/1995), "lo inmediatamente presente representa algo que no está inmediatamente presente, originándose así el significado en sentido psicológico". A esto es lo que llamamos esquema mental: es un proceso totalmente automático que nos lleva, por ejemplo, a que al observar una habitación en la que hay una cama, automáticamente, lo clasifiquemos en nuestra mente como "dormitorio". Por este motivo, dicho autor defendía que gran parte de lo que llamamos percepción es, en realidad, recuerdo. Cuando un individuo observa una escena y más tarde debe recordarla, tiende a dar más información de la que realmente percibió. En opinión de Bartlett, el observador rellena las lagunas ayudándose de lo que en situaciones similares ha vivido o con aquello que encaja mejor con la situación.

Por ello, como ya demostraron Brewer y Treyens en 1981 en su investigación sobre este tema, los esquemas juegan un papel muy importante en la memoria. Los esquemas matizan lo que se recuerda al favorecer la formación de estereotipos y sirven para completar la información que falta o extraer inferencias. Pueden influir en el contenido de la memoria a largo plazo y también en cómo se recupera la información, haciendo que tendamos a recordar sólo lo necesario en el momento de la recuperación (Morris \& Maisto, 2001). Sin embargo, el modo en el que se introducen estas falsas memorias en los esquemas mentales aún está en debate (debido, sobre todo, a lo poco explorado que está este campo).

Pese a esto, en este estudio se decidió mantener la presentación de los ítems dentro de una imagen debido al realismo que aportaba a la investigación: nuestro entorno no es aséptico, la memoria trabaja sobre una realidad en la que nuestros esquemas están siempre presentes, por lo que se decidió tenerlos en cuenta como un punto más en la investigación, dándole así más profundidad y más cercanía a los datos con la realidad cotidiana.

Se dedujo pues, que ante la presentación de una imagen que sugiriera un esquema mental determinado ("dormitorio" en este caso), los dependientes de campo, al analizar la escena de una manera más holística, mucho 
menos detallada que los independientes de campo, cometerían el "error" de apoyarse más en el esquema mental a la hora de reconstruir el recuerdo que los independientes, lo cual les llevaría a tener más errores de memoria.

En definitiva, la presente investigación aúna las memorias falsas en las categorías asociadas con la dependencia-independencia de campo, teniendo los esquemas mentales como telón de fondo. Se considera que es una propuesta innovadora, puesto que, lo más similar que se ha intentado hasta ahora, ha sido poner la DIC en relación a las memorias falsas con listas de palabras en la investigación de Corson, Verrier y Bucic (2009), encontrando que los participantes dependientes de campo cometían más errores de memoria que los participantes independientes de campo.

Usando de base las conclusiones de ese estudio, se hipotetizó que en el campo visual también se encontrarían más memorias falsas en los dependientes de campo que en los independientes. Así mismo, se consideró que, al ser el estilo cognitivo DIC un continuo, las memorias falsas también se presentarían en forma de continuo. Para demostrar esto, se analizó también a la muestra intermedia para comprobar si se situaban en un punto medio en cuanto a memorias falsas entre los dependientes y los independientes de campo. Y, por último, se analizó si se producían más memorias falsas con los ítems pertenecientes a categorías que con los ítems independientes.

\section{MÉTODO}

\section{a. Participantes}

Los participantes fueron un total de 214 , con edades comprendidas entre 15 y 50 años $(M=24,73, S D=8,21)$. Se utilizó como criterio de exclusión no padecer trastornos visoespaciales, puesto que se encontró que algunas personas con dislexia mostraban dificultades a la hora de realizar el test de dependencia-independencia de campo. De esta manera, se redujo la muestra a 209 participantes.

Dado que la dependencia y la independencia de campo se corresponden respectivamente con un $15 \%$ de la población, es lógico que tan sólo se hallaran 32 participantes dependientes de campo y 25 participantes independientes de campo en el total de la muestra. Del resto de participantes, se seleccionaron al azar 28 sujetos con una puntuación intermedia en el test para representar a la muestra intermedia y tener así tres grupos equiparables.

Finalmente, sólo 85 participantes (38 hombres, 47 mujeres) cumplían los requisitos para la participación en el estudio, distribuidos como se recoge en la Tabla 1.

Tabla 1. Distribución de la muestra por sexo y DIC

\begin{tabular}{cccc}
\hline \hline & \multicolumn{4}{c}{ Distribución de los participantes según DIC } \\
\hline \hline Sexo & DC & Intermedio & IC \\
\hline Hombre & 14 & 14 & 10 \\
\hline Mujer & 18 & 14 & 15 \\
\hline \hline
\end{tabular}

\section{b. Materiales}

Para la presente investigación se utilizaron las siguientes pruebas:

- Breve cuestionario acerca del sexo, edad y dislexia/trastornos visoespaciales.

- El test de Formas Ocultas: dependencia-independencia de campo (FO), de Seisdedos Cubero (1990).

- Una prueba de memoria consistente en una lámina con una escena de un dormitorio infantil y una plantilla de respuesta de $6 \times 6$ en la que aparecen 12 elementos presentes en la escena ( 4 muebles, 4 juguetes, 4 elementos de papelería) y 24 elementos distractores: 12 distractores de las mismas tres categorías y 12 distractores sin categoría grupal.

\section{c. Diseño}

Esta investigación se trata de un diseño cuasiexperimental con una variable independiente y dos variables dependientes.

- Variable independiente: La dependenciaindependencia de campo es una variable cuantitativa continua, pero, al clasificar las puntuaciones por rangos se convierte en cualitativa nominal con tres niveles: dependiente de campo (0-30), muestra intermedia (40-60) e independiente de campo (70-100).

- Variables dependientes: Ambas son cuantitativas discretas; son la tasa de aciertos y la tasa de falsas alarmas de los participantes en la prueba de memoria. 


\section{d. Procedimiento}

En primer lugar, se pedía a los participantes que rellenaran el formulario de la primera página de la tarea con sus datos personales requeridos (edad, sexo y posibles problemas visoespaciales); y, a continuación, se presentaba la lámina con la escena que debían visualizar durante 20 segundos. Se trataba del dibujo de un dormitorio infantil con 43 elementos dispersos por la habitación (ver Figura 1).

Figura 1. Lámina de la tarea de memoria

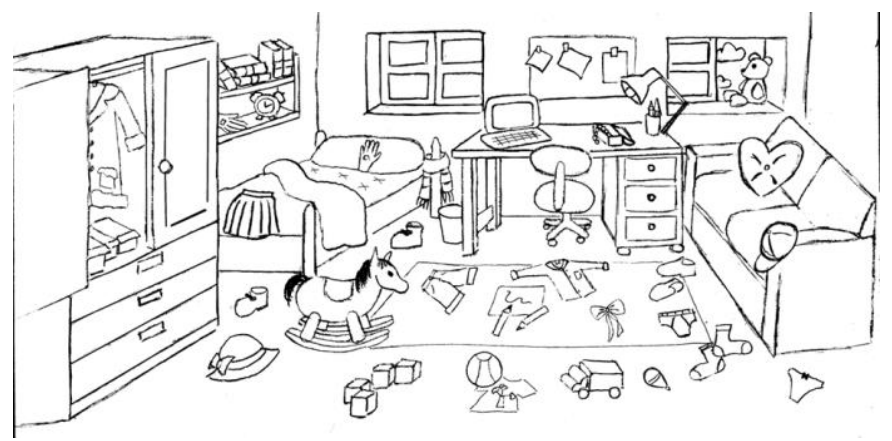

La tarea de reconocimiento tiene lugar entre diez y quince minutos después, tras una tarea con suficiente carga cognitiva que aseguraba que ni la memoria operativa ni a corto plazo intervendrían en el recuerdo. Dicha tarea era el test de Formas Ocultas, que requería seis minutos para su realización y entre cinco y diez previos para la explicación de la tarea.

El test de Formas Ocultas permitió dividir a los participantes en tres categorías:

- 0 - 30: Dependientes de campo (DC).

- 40 - 60: Muestra intermedia (MI).

- 70 - 100: Independientes de campo (IC).

Las clasificaciones de dependencia e independencia de campo son las originales de Witkin (1987), siendo excluidas de la distribución las franjas contiguas para diferenciar mejor los grupos. Todos los participantes que no encajaban dentro de estas categorías $(30-40 ; 60-70)$ eran rechazados.

Tras realizar este test, se procedía a cumplimentar la plantilla de respuestas de la prueba de memoria visual (Figura 2) consistente en 36 imágenes sin color presentadas en un formato $6 \times 6$, de las cuales, sólo 12 estaban presentes en la lámina original que visualizaron los participantes y las otras 24 eran distractores: 12 que no se agrupaban en categorías y 12 de las mismas tres categorías que los elementos sí presentes (juguetes, papelería y muebles).

Figura 2. Plantilla de respuesta para la tarea de memoria.
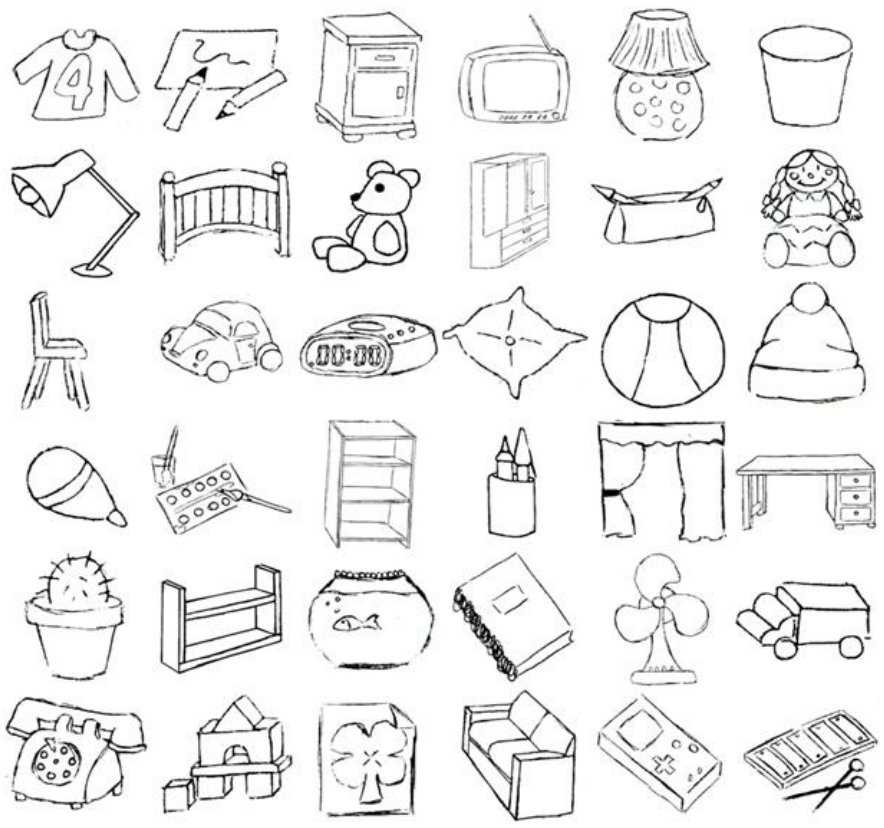

Los ítems de esta tarea están clasificados en la Tabla 2, organizados por su pertenencia o no a una categoría y separando los distractores de los elementos que sí estaban presentes en la Figura 1.

De los elementos estudiados, se pueden encontrar seis de cada categoría en la imagen pero sólo se pedía el recuerdo de cuatro de cada una de esas categorías (los marcados con *).

Tabla 2. Tipos de ítems en la tarea de memoria

\begin{tabular}{|c|c|c|c|}
\hline & Juguetes & Papelería & Muebles \\
\hline \multirow{6}{*}{$\begin{array}{c}4 \text { presentados } \\
(\text { sólo los } *)\end{array}$} & Caballo & Corcho & Armario* \\
\hline & Camión* & Dibujo* & Cama \\
\hline & Cubos & Flexo* & Escritorio* \\
\hline & Osito* & Libros & Estantería* \\
\hline & Pelota* & Papelera* & Silla \\
\hline & Peonza* & Portalápices* & Sofá* \\
\hline \multirow{5}{*}{$\begin{array}{c}4 \text { distractores } \\
\text { de } \\
\text { misma } \\
\text { categoría }\end{array}$} & Cochecito & \multirow{5}{*}{$\begin{array}{c}\text { Acuarelas } \\
\text { Estuche } \\
\text { Libreta } \\
\text { Póster }\end{array}$} & Cabecero \\
\hline & Cubos de & & Estantería \\
\hline & construcción & & grande \\
\hline & Muñeca & & Mesilla \\
\hline & Xilófono & & Silla \\
\hline \multirow{4}{*}{$\begin{array}{l}12 \text { distractores } \\
\text { sin categoría }\end{array}$} & Cojín & \multirow{4}{*}{$\begin{array}{l}\text { Cortinas } \\
\text { Jersey } \\
\text { Planta } \\
\text { Ventilador }\end{array}$} & Despertador \\
\hline & Gorro de lana & & digital \\
\hline & Pecera & & Lampara \\
\hline & Televisión & & $\begin{array}{l}\text { Videojuego } \\
\text { Videno }\end{array}$ \\
\hline
\end{tabular}

Se pedía a los participantes que marcaran los elementos que recordaran haber visto en la lámina, sin pedir ni 
mínimo ni máximo, y sin tiempo límite. La tarea de reconocimiento constaba únicamente de imágenes y no de preguntas (p.ej.: ¿había una pelota?) para tratar de evitar al máximo la interferencia producida por el investigador y excluyendo cualquier tipo de interferencia verbal.

La duración total del experimento era entre 12 y 15 minutos.

\section{RESULTADOS}

Para el análisis de los resultados se utilizó el programa estadístico IBM SPSS Stadistics 19. En primer lugar, se calcularon las medias de aciertos y de falsas alarmas para cada uno de los tres grupos (DC, IC y MI), que pueden observarse en la Gráfica 1.

Gráfica 1. Tasa de aciertos y falsas alarmas de los participantes en la prueba de memoria

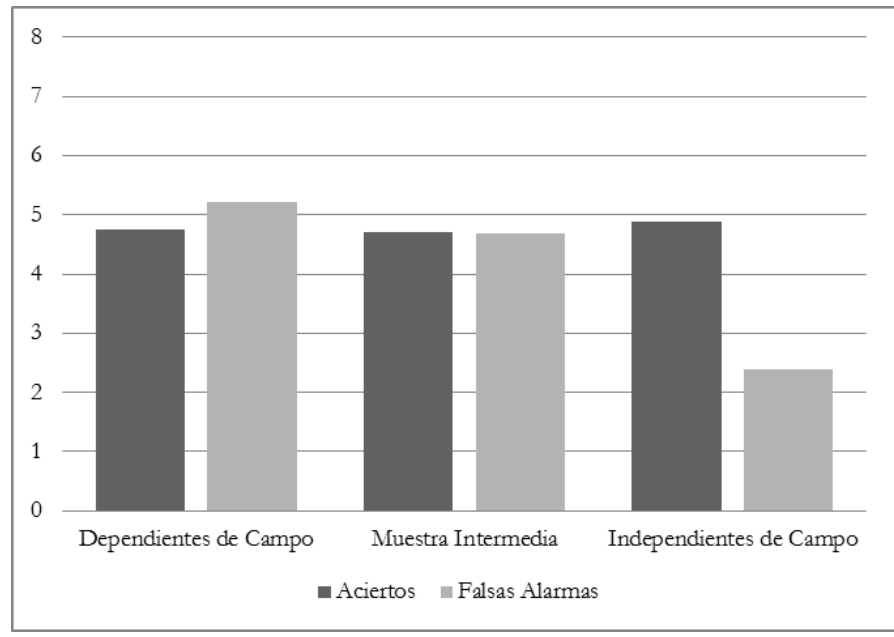

Se utilizó la prueba H de Kruskal-Wallis para poner en relación la tasa de aciertos y falsas alarmas con el DIC. Se encontró que no hay diferencias significativas entre los tres grupos en cuanto a aciertos $\left(\chi^{2}(2)=0,024, \mathrm{p}=0,988\right)$, pero sí respecto a falsas alarmas $\left(\chi^{2}(2)=14,524, \mathrm{p}=0,001\right)$.

Tabla 3. Medias y desviaciones típicas de aciertos y falsas alarmas

\begin{tabular}{cccc}
\hline \hline \multirow{3}{*}{ Aciertos } & & Media & DT \\
\cline { 2 - 4 } & DC & 4,75 & 2,08 \\
\cline { 2 - 4 } & MI & 4,71 & 1,74 \\
\hline \hline \multirow{3}{*}{ Falsas alarmas } & IC & 4,88 & 2,35 \\
\cline { 2 - 4 } & DC & 5,21 & 2,86 \\
\cline { 2 - 4 } & MI & 4,68 & 3,23 \\
\hline \hline
\end{tabular}

Tras demostrar que la muestra cumple la normalidad mediante la prueba de Kolmogorov-Smirnov ( $\mathrm{p}=0,151)$, se procedió entonces a calcular Bonferroni para determinar entre qué grupos se daban las diferencias en falsas alarmas. Se encontró una diferencia significativa entre el grupo intermedio y el independiente de campo $(p=0,013)$ y entre el dependiente y el independiente de campo $(\mathrm{p}=0,001)$. Por lo tanto, los datos concuerdan con la primera hipótesis.

Para contrastar la segunda hipótesis, si la presencia de categorías en los distractores afecta a las falsas memorias, se realizó una prueba $\mathrm{T}$ para muestras relacionadas para comparar la tasa de falsas alarmas en los dos tipos de distractores de la prueba de memoria: los distractores de categoría y distractores sin categoría. Se encontró que no había diferencias significativas entre la tasa de ambos tipos de falsas alarmas $(t=1,467, p=0,146)$.

Posteriormente, se decidió ahondar un poco más en los resultados y buscar si había interacción entre las categorías, tanto en los aciertos como en los distractores, y las categorías DIC. Para esto se realizaron dos ANOVA de dos factores con medidas repetidas para buscar la interacción entre los tres niveles de dependenciaindependencia de campo y las categorías de los ítems (juguetes, papelería y muebles), primero con los aciertos y luego con las falsas alarmas.

En el análisis de los aciertos, tras asumir que la matriz de varianzas-covarianzas es esférica $(p=0,176)$, se puede afirmar que la tasa de recuerdo no es la misma para las tres categorías $(\mathrm{F}(2)=14,285, \mathrm{p}<0,001)$ : de media, los ítems de la categoría muebles eran más recordados que los juguetes y los elementos de papelería. Sin embargo, no se encontró interacción entre la DIC y las categorías en los aciertos $(\mathrm{F}(4)=1,177, \mathrm{p}=0,323)$.

Por otro lado, en el análisis de las falsas alarmas no se puede asumir esfericidad ( $\mathrm{p}=0,008)$, por lo que se utilizó el estadístico multivariado Traza de Pillai, con el que se determinó que la tasa de falsas alarmas no es la misma en las distintas categorías $(\mathrm{F}(2)=16,678, \mathrm{p}<0,001)$, dado que de media, los ítems distractores de papelería producían menos memorias falsas que los de juguetería y muebles; y además, en este caso sí había interacción con el DIC $(\mathrm{F}(4)=3,199, \mathrm{p}=0,015)$. Esto se debe a que la categoría papelería es escogida con más frecuencia por la muestra intermedia que por los dependientes o los independientes de campo, siendo la única categoría que no cumple el supuesto de progresión lineal de aumentar cuanto más dependientes de campo son los participantes, como podemos ver en la Gráfica 2.

Gráfica 2. Interacción entre el DIC y las FFAA por categorías 


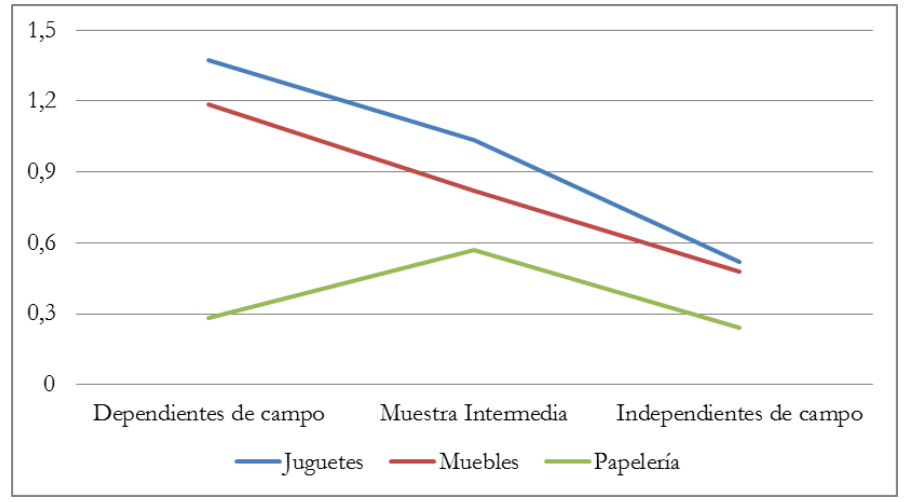

Para completar la investigación, se analizó cada ítem por separado para ver la tasa de elección de los participantes según el DIC. No se aprecian diferencias significativas salvo para cuatro ítems: el sofá, los cubos de construcción, la mesilla y las acuarelas.

El sofá $\left(\chi^{2}=8,977, \mathrm{p}=0,011\right)$ es recordado por el grupo de dependientes de campo sólo en el 31,25\% de los casos, mientras que los independientes de campo lo recuerdan el $60 \%$ y la muestra intermedia el $67,86 \%$.

Respecto a los ítems distractores, los cubos de construcción $\left(\chi^{2}=6,735, \mathrm{p}=0,034\right)$ son recordados por el $37,5 \%$ de los dependientes de campo y el $32,14 \%$ de la muestra intermedia, pero tan sólo por un $8 \%$ de los independientes de campo. Algo parecido ocurre con el distractor mesilla $\left(\chi^{2}=8,272, \mathrm{p}=0,016\right)$, que es recordado por el $40,63 \%$ de los dependientes de campo y el $21,43 \%$ de la muestra intermedia, pero sólo un $8 \%$ de independientes de campo decía recordarlo.

El único dato discordante es el del distractor acuarelas $\left(\chi^{2}=10,815, p=0,004\right)$, elegidas por la muestra intermedia en el $17,86 \%$ de las veces, y en ninguna ocasión por los independientes y dependientes de campo.

Por último, se analizó qué ítems, dentro de los distractores, habían sido los más y los menos seleccionados a nivel general.

Tabla 4. Tasa de los elementos menos y más seleccionados

\begin{tabular}{cccc}
\hline \hline \multicolumn{2}{c}{ Menos seleccionados } & \multicolumn{2}{c}{ Más seleccionados } \\
\hline Ítem & $\%$ de selección & Ítem & $\%$ de selección \\
\hline \hline Planta & $8 \%$ & Cabecero & $32 \%$ \\
\hline Televisor & $8 \%$ & Cortinas & $25 \%$ \\
\hline Ventilador & $8 \%$ & Mesilla & $25 \%$ \\
\hline Videojuego & $7 \%$ & Muñeca & $35 \%$ \\
\hline \hline
\end{tabular}

Como se puede observar, hay una tasa más baja de errores de memoria en los objetos que menos encajan con el esquema de "dormitorio infantil" como lo son un televisor, un ventilador, una planta o un videojuego. Sin embargo, los elementos que mejor encajan en este esquema como las cortinas, la mesilla, el cabecero o la muñeca (Tabla 4) reciben una tasa de elección más alta. Esto se debe a que, cuando los distractores son más típicos o representativos de las escenas, se podrá esperar mayor tasa de errores de memoria (Brewer y Treyens, 1981).

\section{DISCUSIÓN}

Como se ha podido comprobar en los resultados, la primera hipótesis recibe aval empírico: la dependenciaindependencia de campo influye en la formación de errores de memoria.

Como demostró el experimento de Emmett (2003), el entorno ayuda a la codificación de los recuerdos en los dependientes de campo y, en ausencia de éste, su recuerdo es menor. Por lo que se puede deducir que, al hacer estudiar a los participantes la habitación como un "todo", en lugar de presentar los elementos de manera serial y descontextualizados, y al tener que recordar sus elementos por separado, la tarea se les plantea más difícil que a los independientes de campo, a los que apenas afecta el entorno en la codificación. Al no disponer del entorno como ayuda mnemónica, los dependientes de campo recurren a su esquema de "dormitorio" para completar la tarea de reconocimiento.

Teniendo en cuenta las ideas expuestas hasta ahora, se podría concluir que los independientes de campo cometen menos errores de memoria que los dependientes de campo debido a que recurren menos a sus esquemas mentales para completar sus recuerdos. Así mismo, se puede decir que esto no afecta a la tasa de aciertos, sino a las falsas alarmas, es decir, al hecho de añadir información a la ya existente.

Basándose en esto, se pueden justificar los resultados significativos de los ítems señalados en el apartado anterior. El ítem "sofá" no encaja en el esquema "dormitorio", por este motivo es recordado mucho más por los independientes de campo que podrían realmente recordar el ítem, y no por los dependientes, que estarían realizando una observación menos detallada y más holística basada en el esquema. Así mismo, los ítems distractores "mesilla" y "cubos de construcción" son elegidos con más 
frecuencia por los dependientes de campo que por los independientes, puesto que sí encajan en el esquema y resultan similares a otros objetos que sí estaban presentes en la lámina.

Respecto al segundo objetivo del estudio, comprobar si había más falsas memorias en los ítems pertenecientes a las categorías que en los ítems independientes, todo parece indicar que, aunque la tasa de falsas alarmas producida por los distractores de categoría es ligeramente superior a la de distractores genéricos, no hay diferencia significativa entre éstos.

Esto podría ser debido a que, al estudiar los elementos dentro de una imagen, esto podría llevar a que el participante se ayude del esquema de dicha imagen y no analice los elementos por separado. Por este motivo no se habría podido producir el efecto de categorías asociadas, dado que se estudiaban como parte de un todo y, los participantes, no tenían la necesidad de asociarlos mentalmente para codificarlos de manera más sencilla.

Otra explicación plausible podría ser que, al igual que en el experimento de Hintzman (1998) que se comentaba en la introducción, la cantidad de ítems presentados de cada categoría haya sido demasiado pequeña como para producir el efecto de categorías. En una futura investigación esto habría de tenerse en cuenta a la hora de seleccionar el número de ítems.

Por otra parte, dado que el estilo cognitivo DIC es un continuo, como ya se ha mencionado previamente, es lógico pensar que su progresión en cuanto a errores de

\section{V.REFERENCIAS}

Arnau Gras, J., Blanca Mena, M.J. \& Salvador Beltrán, F. (1992). Diferenciación hemisférica, estilos cognitivos y procesamiento de la información visual. Psicothema, 4, 237-252.

Bartlett, F.C. (1995). Recordar. Estudio de psicología experimental y social. Madrid: Alianza Editorial. (Original: Bartlett, F.C. (1932). Remembering: A Study in Experimental and Social Psychology. Cambridge: Cambridge University Press)

Brewer, W.F. \& Treyens, J.C. (1981). Role of Schemata in Memory for Places. Cognitive Psychology, 13, 207-230.

Corson, Y., Verrier, N. \& Bucic, A. (2009). False memories and individual variations: The role of Field Dependence-Independence. Personality and Individual memoria pudiera ser lineal. Podría considerarse entonces, que ese debería hacer sido el resultado encontrado y no un grupo de muestra intermedia tan cercano a la dependencia de campo en cuanto al rendimiento. Se podría achacar este resultado a la elección de ítems en la categoría papelería que, como se puede observar en los resultados, no siguieron la progresión lineal de las categorías juguetes y muebles, que sí se ajustan a los resultados esperados, y además producía pocas memorias falsas en los participantes. Los ítems de papelería bajaron la media de errores de los dependientes de campo y aumentaron la de la muestra intermedia, equiparando ambas. Podría considerarse que ha sido un problema en cuanto a la elección de los ítems distractores de la categoría elementos de papelería, puesto que las otras dos categorías sí cumplen el supuesto. Bajo este enfoque sí se podría afirmar que la tasa de falsas alarmas es una progresión lineal.

En conclusión, aunque la muestra no ha podido ser muy cuantiosa dado que los participantes que cumplieran los requisitos DIC eran difíciles de encontrar por su propia naturaleza de ser extremos poblacionales, los resultados han sido satisfactorios. Este estudio ofrece una base sólida para una futura investigación, con la expectativa de que la influencia de la dependencia-independencia de campo en las memorias falsas podrá ser probada una vez más en el futuro con resultados favorables, añadiendo un nuevo elemento a tener en cuenta en la psicología del testimonio.

\section{Differences, 47, 8-11.}

Emmett, D., Clifford, B.R. \& Gwyer, P. (2003). An investigation of interaction between cognitive style and context reinstatement on the memory performance of eyewitnesses. Personality and Individual Differences, 34, 1495-1508.

Fisher, A.V. \& Sloutsky, V.M. (2004). Categorization and memory: Representation of category information increases memory intrusions. In K. Forbus, D. Gentner, \& T. Regier (Eds.), Proceedings of the XXVI Annual Conference of the Cognitive Science Society, 387391 Mahwah, NJ: Erlbaum.

Hintzman, D.L. (1988). Judgments of frequency and recognition memory in a multiple-trace memory 
model. Psychological Review, 95, 528-551.

Morris, C.G. \& Maisto, A.A. (2001). Introducción a la Psicología. México: Pearson Educación.

Seisdedos, N. (1990). Formas Ocultas: DependenciaIndependencia de campo. Madrid: Tea Ediciones.

Strack, F. \& Bless, H. (1994). Memory for nonoccurrences: Metacognitive and presuppositional strategies. Journal of Memory and Language, 33, 203-217.
Innocence Project. (2016). Eyewitness misidentification. Recuperado a 27 de abril de 2016, de http://www.innocenceproject.org/causes/eyewitne ss-misidentification/

Witkin, H.A. \& Goodenough, D.R. (1985). Estilos cognitivos. Naturaleza y orígenes. Madrid: Ediciones Pirámide.

Witkin, H. A., Oltman, P. K., Raskin, E. \& Karp, S. A. (1987). EFT, GEFT, CEFT, Test de figuras enmascaradas. Madrid: Tea Ediciones. 